\title{
A Robust Adaptive Controller for Robot Manipulators
}

\author{
Harry Berghuis $\left(^{*}\right)$, Romeo Ortega $\left(^{* * *}\right)$, Henk Nijmeijer $(* *) \cdot$ \\ $\left(^{*}\right)$ Department of Electrical Engineering, $\left({ }^{* *}\right)$ Department of Applied Mathematics \\ University of Twente, P.O. Box 217, 7500 AE Enschede, Netherlands. \\ (***) Department of Electrical Engineering \\ McGill University, 3480 University Street, Montréal, Québec, H3A 2A7, Canada.
}

\begin{abstract}
In the present paper we propose a globally convergent adaptive control scheme for robot motion control with the following features: First, the adaptation law possesses enhanced robustness with respect to noisy velocity measurements. Secondly, the controller does not require the inclusion of high gain loops that may excite the unmodeled dynamics and amplify the noise level. Thirdly, we derive for the known parameter design a relationship between compensator gains and closed-loop convergence rates which is independent of the robot task. This helps the designer to carry out the gain tuning with an eye on the robustness - performance tradeoff.
\end{abstract}

Keywords - Robot systems, Adaptive control, Robustness, Lyapunov stability.

\section{Introduction}

The path tracking control problem of rigid robots with uncertain parameters that attracted the attention of robot control theorists in the last few years has matured to a stage where theoretically satisfactory asymptotic results are now well established, see e.g. Ortega and Spong (1989). In order for these results to penetrate the realm of applications there are at least three basic requirements that should be satisfied. First, the adaptation law should not be sensitive to (unavoidable) velocity measurement noise. Secondly, high gain designs, that excite the unmodeled torsional modes and aggravate the noise sensitivity problem (Nicosia and Tomei, 1990), should be avoided. Thirdly, nonconservative measures to carry out the gain tuning taking into account the closed-loop robustness-performance tradeoff should be provided to the designer. In particular, it is desirable to have available relationships between controller gain ranges and convergence rate bounds which to some extent are independent of the specific task. To the best of our knowledge, all existing adaptive controllers for which global stability of the closed-loop can rigorously be proven fail to satisfy all of the requirements mentioned above. Some representative examples are briefly discussed below.

Probably the most elegant solution to the adaptive motion control problem is provided by the so-called passivity based methods, e.g. Slotine and Li (1987) and Sadegh and Horowitz (1987). An important drawback of these schemes is that they are not robust to velocity measurement noise. Specifically, in underexcited operation, e.g. when performing a regulation task where the desired trajectory has become constant, the well known phenomenon of parameter drift (Sastry and Bodson, 1988) in the adaptation law is prone to occur due to the presence of quadratic terms in the measured velocity. This phenomenon has been illustrated in simulations (Sadegh and Horowitz, 1990; Schwartz et al., 1990; Berghuis et al., 1991) and actual experimentation (Ghorbel et al., 1990; Leahy and Whalen, 1991).

A number of adaptive schemes that do not suffer from the velocity measurement problem were recently proposed by Bayard and Wen (1988). However, these schemes require high controller gains in order to both overcome the uncertainty in the initial parameter errors and compensate for the dependency on the magnitude of the desired trajectory velocity.

An alternative approach to obtain enhanced robustness for noise was presented by Sadegh and Horowitz (1990), who propose to replace the actual position and velocity in the regressor by the desired trajectory values. This modification brings along two new difficulties: the inclusion of an additional feedback proportional to the square of the tracking error, that may induce a high gain loop during the transients, and also a lower bound on the compensator gains that is dependent on the magnitude of the desired trajectory velocity. This bound translates again into a high gain requirement when tracking fast reference signals. Berghuis et al. (1991) remove the latter restriction on the 
controller gains but still require the nonlinear proportional feedback in order to be able to show global convergence.

The clever inclusion of of a normalization term in the parameter adaptation law (as well as in the Lyapunov function) allows Whitcomb et al. (1991) to establish global stability for an adaptive scheme without the parameter drift problem nor the need for the nonlinear proportional feedback term, but still requiring the controller gains to satisfy an inequality that depends on the desired trajectory velocity. As we will show below, this condition translates into a task-dependent upper bound on the attainable convergence rates.

The main contribution of this paper is to combine ideas of Berghuis et al. (1991) and Whitcomb et al. (1991) to come up with an adaptive controller that is robust with respect to velocity measurement noise, does not require high gain loops and to provide a relationship between convergence rates and compensator gains that is independent of the desired trajectory velocity magnitude. Furthermore the required additional computations are basically negligible.

The remaining part of the paper is organized as follows. For clarity we have separated into the known and unknown parameter case. Our main results conceming the non-adaptive controller are presented in section 2 . The adaptive case is presented in section 3 . We will present some conclusions in section 4.

\section{Known Parameter Case}

A. Main result

Consider a standard $n$-degree of freedom rigid robot model of the form (Spong and Vidyasagar, 1989)

$$
M(q) \ddot{q}+C(q, \dot{q}) \dot{q}+G(q)=\tau, \quad q \in R^{\mathrm{n}}
$$

where $C(q, \dot{q})$ is defined using the Christoffel symbols. Let the control torque $\tau$ be given as

$$
\tau=M(q) \ddot{q}_{\mathrm{d}}+C(q, \dot{q}-\lambda e) \dot{q}_{\mathrm{d}}+G(q)-K_{\mathrm{d}} \dot{e}-K_{\mathrm{p}} e
$$

with $e \equiv q-q_{\mathrm{d}}, q_{\mathrm{d}} \in R^{\mathrm{n}}$ represents a desired trajectory, and $K_{\mathrm{d}}=K_{\mathrm{d}}^{\mathrm{T}}>0, K_{\mathrm{p}}=K_{\mathrm{p}}^{\mathrm{T}}>0$. Furthermore

$$
\lambda \equiv \frac{\lambda_{0}}{1+\|e\|}
$$

with $\lambda_{0}$ a positive constant, and $\|$. $\|$ is defined as the Euclidean norm.

Assume the controller gains are chosen such that

$$
\lambda_{0}<\min \left\{\frac{K_{\mathrm{d}, \mathrm{m}}}{\left(3 M_{\mathrm{M}}+2 C_{\mathrm{M}}\right)}, \frac{4 K_{\mathrm{p}, \mathrm{m}}}{\left(K_{\mathrm{d}, \mathrm{M}}+K_{\mathrm{d}, \mathrm{m}}\right)}\right\}
$$

where $K_{\mathrm{d}, \mathrm{m}} \equiv \sigma_{\mathrm{m}}\left(K_{\mathrm{d}}\right), K_{\mathrm{d}, \mathrm{M}} \equiv \sigma_{\mathrm{M}}\left(K_{\mathrm{d}}\right), K_{\mathrm{p}, \mathrm{m}} \equiv \sigma_{m}\left(K_{\mathrm{p}}\right)$, with $\sigma_{\mathrm{m}}(),. \sigma_{\mathrm{M}}($.$) the minimum and maximum singular$ value respectively, and $M_{\mathrm{m}}, M_{\mathrm{M}}$ and $C_{\mathrm{M}}$ satisfy (cf. Craig, 1988)

$$
\begin{aligned}
& 0<M_{\mathrm{m}} \leq\|M(q)\| \leq M_{\mathrm{M}} \\
& \|C(q, x)\| \leq C_{\mathrm{M}}\|x\| \text { for all } x
\end{aligned}
$$

Then we can prove the following result.

Proposition 2.1. Under the condition (2.4), the closedloop system is globally convergent, that is e and $\dot{e}$ asymptotically converge to zero and all internal signals are bounded. If besides (2.4) the condition

$$
\lambda_{0}^{2}<\frac{4 M_{\mathrm{m}} K_{\mathrm{p}, \mathrm{m}}}{M_{\mathrm{M}}^{2}}
$$

holds, then the closed-loop system is globally exponentially stable, that is, there exist $m>0, \rho>0$, independent of the desired trajectory velocity, such that

$$
\|x(t)\|^{2} \leq m e^{-p t}\|x(0)\|^{2} \text { for all } t \geq 0
$$

where $x^{\mathrm{T}}=\left[e^{\mathrm{T}} \dot{e}^{\mathrm{T}}\right]$.

Proof. We will strongly rely on the following well known properties of $C(q,$.

$$
\begin{aligned}
& C(q, x) y=C(q, y) x \\
& C(q, x+\alpha y)=C(q, x)+\alpha C(q, y)
\end{aligned}
$$

for all $x, y, q \in R^{\mathrm{n}}, \alpha \in R$.

Combining (2.1) and (2.2) and using (2.8b) we obtain

$$
M(q) \ddot{e}+C(q, \dot{q}) \dot{e}+\lambda C(q, e) \dot{q}_{\mathrm{d}}+K_{\mathrm{d}} \dot{e}+K_{\mathrm{p}} e=0
$$

Consider the positive definite Lyapunov function candidate

$$
V(e, \dot{e})=\frac{1}{2} s^{\mathrm{T}} M(q) s+\frac{1}{2} e^{\mathrm{T}} K_{\mathrm{p}} e
$$

where

$$
s=\dot{e}+\lambda e
$$


With abuse of notation we will write $V(e, \dot{e})$ everywhere although we may change the coordinates $(e, \dot{e})$ into other combinations.

Taking the time-derivative of (2.10) along the trajectory of (2.9) yields

$$
\begin{aligned}
\dot{V}(e, \dot{e})= & s^{\mathrm{T}}[\lambda M(q) \dot{e}+\dot{\lambda} M(q) e+\lambda C(q, \dot{q}) e- \\
& \left.-\lambda C(q, e) \dot{q}_{\mathrm{d}}-K_{\mathrm{d}} \dot{e}-K_{\mathrm{p}} e\right]+\dot{e}^{\mathrm{T}} K_{\mathrm{p}} e
\end{aligned}
$$

where we have used $(2.8 b)$ and the skew-symmetry of $\dot{M}(q)-2 C(q, \dot{q})$ (Ortega and Spong, 1989). Now, (2.8a) allows us to rewrite (2.12) as

$$
\begin{aligned}
\dot{V}(e, \dot{e})= & -s^{\mathrm{T}}\left[K_{\mathrm{d}}-\lambda M(q)\right] \dot{e}+\dot{\lambda} s^{\mathrm{T}} M(q) e+ \\
& +\lambda s^{\mathrm{T}} C(q, \dot{e}) e-\lambda e^{\mathrm{T}} K_{\mathrm{p}} e
\end{aligned}
$$

At this moment we introduce a new variable that will simplify our further developments, namely

$$
s_{1} \equiv \dot{e}+\frac{\lambda}{2} e
$$

In terms of $s_{1}$ we can rewrite (2.13) as

$$
\begin{aligned}
\dot{V}(e, \dot{e})= & -s_{1}^{\mathrm{T}}\left(K_{\mathrm{d}}-\lambda M(q)\right) s_{1}+\dot{\lambda}_{s}^{\mathrm{T}} M(q) e+\lambda s^{\mathrm{T}} C(q, \dot{e}) e \\
& -\left(\frac{\lambda}{2} e\right)^{\mathrm{T}}\left[4 \lambda^{-1} K_{\mathrm{p}}-\left(K_{\mathrm{d}}-\lambda M(q)\right)\right]\left(\frac{\lambda}{2} e\right)
\end{aligned}
$$

In the Appendix we establish the following bounds for the second and third term at the right hand side

$$
\begin{aligned}
& \dot{\lambda}_{s} s^{\mathrm{T}} M(q) e \leq 2 \lambda_{0} M_{M}\left(\left\|s_{1}\right\|^{2}+\left\|\frac{\lambda}{2} e\right\|^{2}\right) \\
& \lambda s^{\mathrm{T}} C(q, \dot{e}) e \leq 2 \lambda_{0} C_{M}\left(\left\|s_{1}\right\|^{2}+\left\|\frac{\lambda}{2} e\right\|^{2}\right)
\end{aligned}
$$

Replacing these bounds in (2.15) and rearranging terms we obtain

$$
\dot{V}(e, \dot{e}) \leq-\kappa_{1}\left\|s_{1}\right\|^{2}-\kappa_{2}\left\|\frac{\lambda}{2} e\right\|^{2}
$$

where

$$
\begin{aligned}
& \kappa_{1} \equiv K_{\mathrm{d}, \mathrm{m}}-3 \lambda_{0} M_{\mathrm{M}}-2 \lambda_{0} C_{\mathrm{M}} \\
& \kappa_{2} \equiv 4 \lambda_{0}^{-1} K_{\mathrm{p}, \mathrm{m}}-K_{\mathrm{d}, \mathrm{M}}-2 \lambda_{0} M_{\mathrm{M}}-2 \lambda_{0} C_{\mathrm{M}}
\end{aligned}
$$

It is easy to see that (2.4) ensures that $\kappa_{1}, \kappa_{2}>0$. Thus $V(e, \dot{e})$ is a non-increasing function bounded from below. This implies from (2.10) that $s, e \in L_{\infty}^{\mathrm{n}}$, and consequently $\dot{e}, s_{1} \in L_{\infty}^{\mathrm{n}}$. Now, because $\lambda \in L_{\infty}$ we conclude from (2.17) that $s_{1}, e \in L_{2}^{\mathrm{n}}$. From square integrability and uniform continuity of $e$ we conclude that it converges to zero. To complete the first part of the proof notice that we also have $\dot{e} \in L_{2}^{\mathrm{n}}$, thus it suffices to establish that $\ddot{e} \in L_{\infty}^{\mathrm{n}}$, which follows from the error dynamics (2.9).

To prove exponential stability let us write $V(e, \dot{e})$ in terms of the coordinates $\left(s_{1}, \frac{\lambda}{2} e\right)$

$$
\begin{aligned}
V(e, \dot{e})= & \frac{1}{2} s_{1}^{\mathrm{T}} M(q) s_{1}+s_{1}^{\mathrm{T}} M(q)\left(\frac{\lambda}{2} e\right)+\frac{1}{2}\left(\frac{\lambda}{2} e\right)^{\mathrm{T}} M(q)\left(\frac{\lambda}{2} e\right) \\
& +\frac{1}{2} e^{\mathrm{T}} K_{p} e
\end{aligned}
$$

which can be bounded as

$$
\begin{array}{r}
\frac{1}{2} \xi_{1}\left\|s_{1}\right\|^{2}+\frac{1}{2} \xi_{2}\left\|\frac{\lambda}{2} e\right\|^{2} \leq V(e, \dot{e}) \leq \\
\leq M_{M}\left\|s_{1}\right\|^{2}+\frac{1}{2} \xi_{3}\left\|\frac{\lambda}{2} e\right\|^{2}
\end{array}
$$

where

$$
\begin{aligned}
& \xi_{1} \equiv M_{\mathrm{m}}-\left(\alpha^{-1} M_{\mathrm{M}}\right)^{2} \\
& \xi_{2} \equiv \frac{4 K_{\mathrm{p}, \mathrm{m}}}{\lambda_{0}^{2}}+M_{\mathrm{m}}-\alpha^{2} \\
& \xi_{3} \equiv \frac{4 K_{\mathrm{p}, \mathrm{M}}}{\lambda^{2}}+2 M_{\mathrm{M}}
\end{aligned}
$$

and $\alpha$ is any positive number.

Under assumption (2.6) we can find $\alpha>0$ such that $\xi_{1}, \xi_{2}>0$. On the other hand, boundedness of $e$ ensures that $\lambda$ is bounded away from zero, and consequently $\xi_{3}<\infty$. From (2.20) and (2.17) we conclude that there exist $m_{1}, \rho>0$ such that

$$
\|y(t)\|^{2} \leq m_{1} e^{-\rho t}\|y(0)\|^{2} \text { for all } t \geq 0
$$

where $y^{T}=\left[\frac{\lambda}{2} e^{T} s_{1}^{T}\right]$. Now we observe that

$$
x=T(\lambda) y
$$

where

$$
T(\lambda)=\left[\begin{array}{cc}
2 \lambda I & 0 \\
-I & I
\end{array}\right]
$$

The proof is completed by noting that

$$
\lambda_{0}>\lambda \geq \lambda_{0}\left(1+\left(\frac{2 V(e(0), \dot{e}(0))}{K_{\mathrm{p}, \mathrm{m}}}\right)^{1 / 2}\right)^{-1}
$$

and consequently $T$ and $T^{-1}$ are bounded matrices. 


\section{B. Discussion}

1. Notice that in contrast to Whitcomb et al. (1991), Sadegh and Horowitz (1990) and Bayard and Wen (1988), the conditions (2.4) and (2.6) on the controller gains $\lambda_{0}, K_{\mathrm{p}}$ and $K_{\mathrm{d}}$ are independent of the desired trajectory velocity. Consequently the convergence rate is also independent of $\dot{q}_{\mathrm{d}}$. This makes the tuning process task independent.

2. It is worth remarking that in the stability proof of the scheme proposed by Whitcomb et al. (1991) a term $\lambda$ (2.3) (denoted $\varepsilon$ in their paper) is introduced in the Lyapunov function. The conditions for stability invoke an upper bound on $\lambda_{0}$ (denoted $\varepsilon_{0}$ in their paper) which depends on $\left\|\dot{q}_{d}\right\|$. Even though $\lambda$ is not used in the (known parameter) control implementation, $\lambda_{0}$ upperbounds the schemes convergence rate, see (13) in Whitcomb et al. (1991), and makes it dependent of the desired trajectory velocity.

3. The proposed control law does not contain a nonlinear PD term as in Sadegh and Horowitz (1990) and Berghuis et al. (1991) which injects to the loop a gain proportional to the square of the tracking error.

4. Two key modifications are introduced in the controller (2.2). The inclusion of an additional term $-\lambda C(q, e) \dot{q}_{\mathrm{d}}$ and the use of the normalization factor $\lambda$. The first idea exploits the structural properties $(2.8)$ of $C(q,$.$) and was introduced$ in Berghuis et al. (1991), while the normalization factor is being used in Whitcomb et al. (1991). The $\lambda$ factor is needed in the controller to be able to bound the cubic term $s^{\mathrm{T}} C(q, \dot{e}) e$ by quadratic terms as done in $(2.16 b)$. Furthermore, the additional term that appears in $\dot{V}(e, \dot{e})$ due to $\dot{\lambda}$ can be upper bounded by quadratic terms in $s_{1}$ and $\frac{\lambda}{2} e$, as shown in (2.16a).

5. To motivate our choice of the Lyapunov function (2.10), which was inspired by Spong et al. (1990), let us consider the one proposed in Whitcomb et al. (1991)

$$
V_{\mathrm{W}}(e, \dot{e})=\frac{1}{2} \dot{e}^{\mathrm{T}} M(q) \dot{e}+\lambda e^{\mathrm{T}} M(q) \dot{e}+\frac{1}{2} e^{\mathrm{T}} K_{\mathrm{p}} e
$$

This function is related to (2.10) by

$$
V_{\mathrm{W}}(e, \dot{e})=V(e, \dot{e})-\frac{1}{2} \lambda^{2} e^{\mathrm{T}} M(q) e
$$

If we evaluate $\dot{V}_{\mathrm{W}}(e, \dot{e})$ we obtain an additional term in $e^{\mathrm{T}} \dot{M}(q) e$. Using the skew-symmetry property this amounts to an extra term in $e^{\mathrm{T}} C(q, \dot{q})$ e. This term can not be compensated by the control and can only be bounded, in terms of $e$ and $\dot{e}$, with a bound on $\dot{q}_{\mathrm{d}}$.

\section{Unknown Parameter Case}

\section{A. Main Result}

Consider the system (2.1) in closed-loop with

$$
\tau=\hat{M}(q) \ddot{q_{\mathrm{d}}}+\hat{C}(q, \dot{q}-\lambda e) \dot{q}_{\mathrm{d}}+\hat{G}(q)-K_{\mathrm{d}} \dot{e}-K_{\mathrm{p}} e
$$

where $\lambda$ is as in (2.3) and

$$
\begin{aligned}
\hat{M}(q) \ddot{q}_{\mathrm{d}}+\hat{C}(q, \dot{q}-\lambda e) \dot{q}_{\mathrm{d}}+\hat{G}(q)= \\
=Y\left(q, \dot{q}-\lambda e, \dot{q}_{\mathrm{d}}, \ddot{q}_{\mathrm{d}}\right) \hat{\theta}
\end{aligned}
$$

with $Y($.$) a regressor matrix that is linear in its second, third$ and fourth argument, and $\hat{\theta}$ a vector of parameters adjusted by

$$
\dot{\hat{\theta}}=-\Gamma Y^{\mathrm{T}}\left(q, \dot{q}-\lambda e, \dot{q}_{\mathrm{d}}, \ddot{q}_{\mathrm{d}}\right) s
$$

where $s$ is given by (2.11).

Proposition 3.1. Assume that (2.4) holds. Then the adaptive system (2.1,3.1-3) is globally convergent, that is e and $\dot{e}$ asymptotically converge to zero and all internal signals are bounded.

Proof. Putting (3.1-2) into (2.1) we obtain

$$
\begin{gathered}
M(q) \ddot{e}+C(q, \dot{q}) \dot{e}+\lambda C(q, e) \dot{q}_{\mathrm{d}}+K_{\mathrm{d}} \dot{e}+K_{\mathrm{p}} e= \\
=Y\left(q, \dot{q}-\lambda e, \dot{q}_{\mathrm{d}}, \ddot{q_{\mathrm{d}}}\right) \tilde{\theta}
\end{gathered}
$$

where

$$
\tilde{\theta}=\hat{\theta}-\theta
$$

and $\theta$ are the true parameters of the robot.

Consider the Lyapunov function candidate

$$
V_{\mathrm{A}}(e, \dot{e}, \tilde{\theta})=V(e, \dot{e})+\frac{1}{2} \tilde{\theta}^{\mathrm{T}} \Gamma^{-1} \tilde{\theta}
$$

with $V(e, \dot{e})$ defined as in (2.10). The time-derivative of $V_{\mathrm{A}}(e, \dot{e}, \tilde{\theta})$ along the error dynamics (3.4) with the choice of the adaptation law (3.3) yields (2.17). Global convergence then follows from the arguments used in the proof of Proposition 2.1.

\section{B. Discussion}

1. The remarks as given in section 2.B also hold for the adaptive case.

2. It is well known (Sastry and Bodson, 1988) that the equilibrium set of adaptive systems is unbounded. There- 
fore, in underexcited conditions and in the presence of noise the instability mechanism of parameter drift appears in the adaptation law. In the present context, excitation is lost in the regulation part of the task, i.e. when $q_{\mathrm{d}}=$ constant. In these circumstances the controller (3.1) reduces to a PD plus adaptive gravitation compensation, and the adaptation law (3.3) with velocity measurement noise $\eta$ looks like

$$
\dot{\hat{\theta}}=-\Gamma Y^{\mathrm{T}}(q, 0,0,0)(\dot{q}+\eta+\lambda e)
$$

On the other hand, the adaptation law of Slotine and $\mathrm{Li}$ (1987) in this situation becomes

$$
\dot{\hat{\theta}}=-\Gamma Y^{\mathrm{T}}\left(q, \dot{q}+\eta,-\lambda_{0} e,-\lambda_{0}(\dot{q}+\eta)\right)\left(\dot{q}+\eta+\lambda_{0} e\right)
$$

Comparing (3.7) and (3.8) we notice that the latter contains quadratic terms in the measurement noise $\eta$, in contrast with (3.7) which only contains linear terms in $\eta$. The integral of these quadratic terms introduces a drift which is proportional to the noise variance (cf. Berghuis et al. (1991)).

3. The adaptation laws presented in Bayard and Wen (1988), Berghuis et al. (1991), Sadegh and Horowitz (1990) and Whitcomb et al. (1991) are for $q_{\mathrm{d}}=$ constant also linear in the measurement noise $\eta$, but these control schemes have the drawbacks as mentioned in the introduction.

4. The extra computations needed in the implementation of the adaptive controller (3.1-3) due to the additional term $-\lambda C(q, e) \dot{q}_{d}$ are negligible. Since $\lambda e$ is already needed in $s$ we only require an extra addition.

5. The scalar gain $\lambda$ is an important design parameter in the adaptation law (3.3). In this respect it is interesting to notice that it is possible to generalize the control schemes (2.2) and (3.1-3) in the sense that $\lambda$ can be chosen to be a diagonal matrix, i.e.

$$
\lambda \equiv \operatorname{diag}\left(\frac{\lambda_{0, \mathrm{i}}}{1+\left\|e_{\mathrm{i}}\right\|}\right), \quad \mathrm{i}=1, \ldots, \mathrm{n}
$$

where $e_{\mathrm{i}}$ is the $\mathrm{i}$-th component of $e$, and $\lambda_{0, \mathrm{i}}>0$. For this choice propositions 2.1 and 3.1 hold if condition (2.4) is replaced by

$$
\lambda_{0, \mathrm{i}}<\min \left\{\frac{K_{\mathrm{d}, \mathrm{m}}}{4 M_{\mathrm{M}}+2 C_{\mathrm{M}}}, \frac{4 K_{\mathrm{p}, \mathrm{m}}}{K_{\mathrm{d}, \mathrm{M}}+K_{\mathrm{d}, \mathrm{m}}}\right\}, \mathrm{i}=1, \ldots, \mathrm{n}(3.10)
$$

and if it is assumed that $K_{\mathrm{p}}$ is diagonal (de Vries, 1992).

\section{Conclusions}

We have presented a globally convergent adaptive control algorithm for robot motion control with enhanced noise sensitivity properties. Moreover, the controller does not contain nonlinear proportional compensation gains and the controller gains and the convergence rate are independent of the desired reference velocity.

To attain this objective we propose a new controller structure which incorporates the normalization idea of Whitcomb et al. (1991) and the additional compensation term of Berghuis et al. (1991). From the analysis point of view, a Lyapunov function similar to the one proposed in Spong et al. (1990) is used to insure negative definiteness of its time-derivative via a suitable change of coordinates. In the non-adaptive case this Lyapunov function allows us to conclude exponential stability with a convergence rate independent of the robot task.

We would like to mention that in order to verify the performance and robustness properties of the proposed control scheme, practical experiments such as the ones presented by Ghorbel et al. (1990) and Whitcomb et al. (1991) need to be performed. Currently we are working on this.

\section{Acknowledgements}

The first author would like to thank the Netherlands Technology Foundation (STW) for supporting his work. The second author would like to thank Dan Koditschek for sending him a preprint of the interesting technical report by Whitcomb et al. (1991). We also would like to thank the Dutch Network on Systems and Control, that financed the stay of the second author at the University of Twente.

\section{References}

Bayard, D.S., and J.T. Wen (1988). New Class of Control Laws for Robotic Manipulators : Adaptive Case. Int. Journal of Control, Vol.47, 1387-1406.

Berghuis, H., P. Löhnberg and H. Nijmeijer (1991). Adaptive 'PD+' Control of Robot Manipulators. Proc. Symp. on Robot Control, Vienna, Austria. To appear.

Craig, J.J. (1988). Adaptive Control of Robot Manipulators. Addison-Wesley, Reading, MA.

Ghorbel, F., A. Fitzmorris and M.W. Spong (1990). Robustness of Adaptive Control of Robots: Theory and Experiment. In Advanced Robot Control, C. Canudas de Wit 
(ed.), Lecture Notes in Control and Information Sciences, Vol. 162, Springer-Verlag, Berlin.

Leahy, M.B. Jr., and P.V. Whalen (1991). Direct Adaptive Control for Industrial Manipulators. Proc. IEEE Conf. on Robotics and Automation, 1666-1672.

Nicosia, S., and P. Tomei (1990). Robot Control by Using Only Joint Position Measurements. IEEE Trans. AC, Vol.35, 1058-1061.

Ortega, R., and M.W. Spong (1989). Adaptive Motion Control of Rigid Robots: a Tutorial. Automatica, Vol.25, 877-888.

Sadegh, N., and R. Horowitz (1987). Stability Analysis of an Adaptive Controller for Robotic Manipulators. Proc. IEEE Int. Conf. on Robotics and Automation, 1223-1229. Sadegh, N., and R. Horowitz (1990). Stability and Robustness Analysis of a Class of Adaptive Controllers for Robotic Manipulators. Int. Journal on Robotics Research, Vol.9, 74-92.

Sastry, S., and M. Bodson (1988). Adaptive Control: Stability, Convergence and Robustness. Prentice-Hall, Englewood Cliffs, NJ.

Schwartz, H.M., G. Warshaw and T. Janabi (1990). Issues in Robot Adaptive Control. Proc. American Control Conference, 2797-2805.

Slotine, J.-J.E., and W. Li (1987). On the Adaptive Control of Robot Manipulators. Int. Journal on Robotics Research, Vol.6, 49-59.

Spong, M.W., R. Ortega and R. Kelly (1990). Comments on "Adaptive Manipulator Control : A Case Study". IEEE Trans. AC, Vol.35, 761-762.

Spong, M.W., and M. Vidyasagar (1989). Robot Dynamics and Control. Wiley, New York.

Vries, D. de (1992). Performance Evaluation of a New Adaptive Controller for Robot Manipulators. Internal Report 92R014, University of Twente, Control Laboratory, Enschede, Netherlands.

Whitcomb, L.L., A.A. Rizzi and D.E. Koditschek (1991). Comparative Experiments with a New Adaptive Controller for Robot Arms. Technical Report 9101, Yale University, Center for Systems Science, New Haven, Connecticut, USA.

\section{Appendix}

Upper bounds for last two right hand side terms in (2.15) are given by

$$
\begin{aligned}
\dot{\lambda} s^{\mathrm{T}} M(q) e & =-\frac{\lambda e^{\mathrm{T}} \dot{e}}{\|e\|(1+\|e\|)} s^{\mathrm{T}} M(q) e \\
& =-\frac{\lambda e^{\mathrm{T}}\left(s_{1}-\frac{\lambda}{2} e\right)}{\|e\|(1+\|e\|)}\left(s_{1}+\frac{\lambda}{2} e\right)^{T} M(q) e \\
& \leq \frac{\lambda M_{M}\|e\|}{1+\|e\|}\left(\left\|s_{1}\right\|^{2}+2\left\|s_{1}\right\|\left\|\frac{\lambda}{2} e\right\|+\left\|\frac{\lambda}{2} e\right\|^{2}\right) \\
& \leq \frac{2 \lambda M_{M}\|e\|}{1+\|e\|}\left(\left\|s_{1}\right\|^{2}+\left\|\frac{\lambda}{2} e\right\|^{2}\right) \\
& \leq 2 \lambda_{0} M_{M}\left(\left\|s_{1}\right\|^{2}+\left\|\frac{\lambda}{2} e\right\|^{2}\right) \\
\lambda s^{\mathrm{T}} C(q, \dot{e}) e & =\lambda\left(s_{1}+\frac{\lambda}{2} e\right)^{T} C(q, e)\left(s_{1}-\frac{\lambda}{2} e\right) \\
& \leq \lambda\|e\| C_{M^{\prime}}\left(\left\|s_{1}\right\|^{2}+2\left\|s_{1}\right\|\left\|_{2}^{\frac{\lambda}{2}} e\right\|+\left\|\frac{\lambda}{2} e\right\|^{2}\right) \\
& \leq 2 \lambda\|e\| C_{M}\left(\left\|s_{1}\right\|^{2}+\left\|\frac{\lambda}{2} e\right\|^{2}\right) \\
& \leq 2 \lambda_{0} C_{M}\left(\left\|s_{1}\right\|^{2}+\left\|\frac{\lambda}{2} e\right\|^{2}\right)
\end{aligned}
$$

